\title{
Der Darm als Angriffsort
}

Neben schweren Symptomen im Bereich der Atemwege weisen bis zu etwa $60 \%$ der Patienten mit COVID-19 auch gastrointestinale Symptome auf. So wurde bei vier bis $34 \%$ von Patienten in China Durchfall festgestellt, in den USA bei zwölf bis $34 \%$. Obwohl eine Schädigung der Darmschleimhaut durch SARS-CoV-2 eine zusätzliche Infektion mit einem gastrointestinalen Keim begünstigen könnte, scheint dies zumindest für non-C. difficile-Pathogene nicht zuzutreffen. Einer zusätzlichen Infektion mit $C$. difficile sollte jedoch besonders angesichts der oft längeren Krankenhausaufenthalte besonderes Augenmerk gewidmet werden, stellt eine Studie aus New York fest.

Der Mechanismus der gastrointestinalen Betroffenheit bei COVID-19 Patienten dürfte über die 2-Rezeptoren, die auf den Epithelzellen des Darms vorhanden sind, zu erklären sein, stellen Laszkowska et al. fest. ACE 2-Rezeptoren sind mittlerweile als Bindungs- und Eintrittsort für Viren gut beschrieben. Darüber hinaus ist es möglich, dass die durch COVID-19 hervorgerufene Entzündung des Darms eine Prädisposition für eine Ko-Infektion mit anderen gastrointestinalen Pathogenen verursacht. Da besonders C. difficile bei bereits schwer erkrankten Personen ein hohes zusätzliches Risiko bedeutet, ist dessen Erkennung und Behandlung ein wichtiger Bestandteil im Therapieverlauf. Angiotensin Converting Enzym (ACE)

\section{C. difficile im Krankeitsverlauf}

Insgesamt, so die Autoren, zeigte sich bei den knapp 5000 Patienten, deren Daten im Beobachtungszeitraum analysiert wurden, dass COVID-19 positive Patienten weniger häufig gastrointestinale Infektionen aufwiesen als COVID-19 negative Patienten. Dies war allerdings auf die Häufigkeit von non-C. difficile Infektionen zurückzuführen. Zwischen COVID-19 positiven und COVID-19 negativen Patienten gab es bei $C$. difficile-Infektionen dagegen keinen signifikanten Unterschied. COVID-19 Patienten mit schwerem Krankheitsverlauf und mit milderem Verlauf wiesen keine signifikanten Unterschiede hinsichtlich der Raten oder Typen gastrointestinaler Pathogene auf. Im Verlauf der Pandemie zeigte sich jedoch zu Beginn in den beiden untersuchten New Yorker

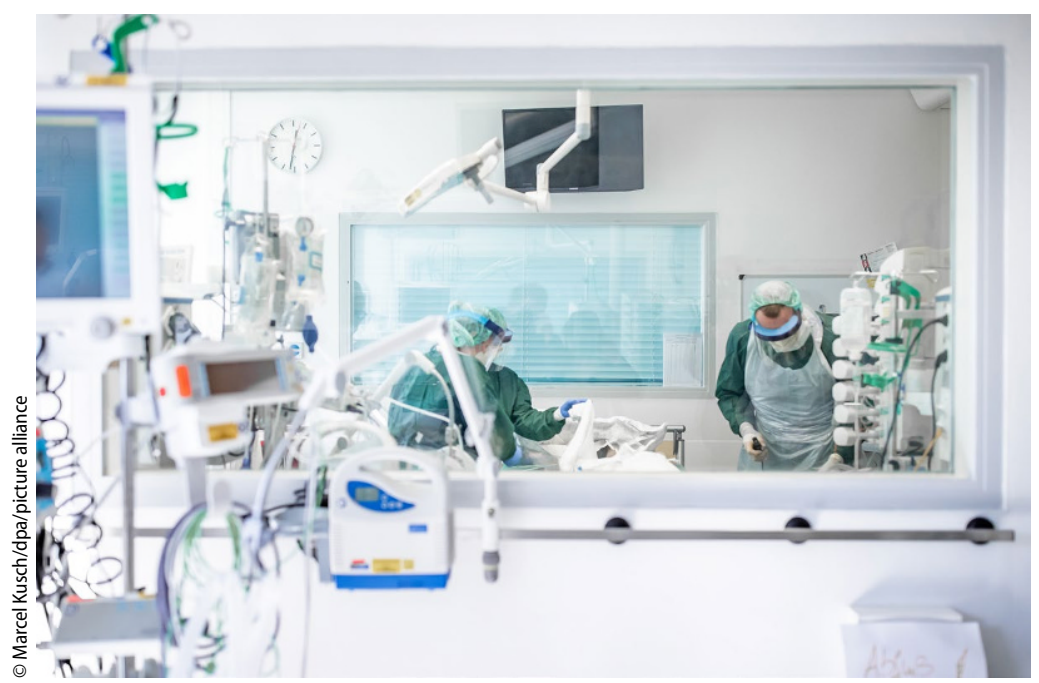

$\Delta$ C. difficile Infektionen sind ein grundsätzliches Problem bei schwer erkrankten Menschen - auch bei COVID-19
Spitälern ein dramatischer Rückgang an Tests auf sowohl $C$. difficile als auch auf nicht- $C$. difficile. Die Testrate auf C. difficile nahm allerdings bald darauf wieder zu. Jene für andere gastrointestinale Pathogene halbierte sich etwa.

C. difficile sollte als mögliche Differenzialdiagnose bei Patienten, die an Diarrhoe leiden, unabhängig von ihrem COVID-19 Status mitbedacht werden, stellen Laszkowska et al. fest. Dies sei vor allem im späteren Verlauf der Erkrankung wichtig, denn auch wenn gastrointestinale Symptome, wie vor allem Diarrhoe, zunächst durch SARS-CoV-2 verursacht werden, gleicht sich das Infektionsprofil über die längere Aufenthaltsdauer im Krankenhaus jenem von Patienten ohne COVID-19 an. Die festgestellte niedrigere Häufigkeit von nichtC. difficile Infektionen bei Patienten mit COVID-19 könnte auch auf die niedrigeren Testraten zurückzuführen sein, geben die Autoren zu bedenken. Hier waren jedenfalls $E$. coli erwartungsgemäß die am häufigsten identifizierten Erreger. Die Identifikation des Krankheitserregers und die zielgerichtete Behandlung stellt einen wichtigen Baustein für die optimale Therapie dar.

Hinweis des Verlags. Der Verlag bleibt in Hinblick auf geografische Zuordnungen und Gebietsbezeichnungen in veröffentlichten Karten und Institutsadressen neutral.

Wien klin Mag 2021 $24: 55$

https://doi.org/10.1007/s00740-02100395-z

(c) Springer-Verlag GmbH Austria, ein Teil von Springer Nature 2021
Quelle: Laszkowska M.: Prevalence of Clostridioides difficile and Other Gastrointestinal Pathogens in Patients with COVID-19, in: Digestive Diseases and Sciences, https:// doi.org/10.1007/ s10620-020-06760ym pub.online 22. Jänner 2021 